\title{
Mutant p53 enhances leukemia-initiating cell self-renewal to promote leukemia development
}

Sarah C. Nabinger ${ }^{1}$, Sisi Chen ${ }^{2}$, Rui Gao ${ }^{1}$, Chonghua Yao ${ }^{1,5}$, Michihiro Kobayashi ${ }^{1}$, Sasidhar Vemula ${ }^{1}$, Aidan C. Fahey ${ }^{1}$, Christine Wang ${ }^{1}$, Cecil Daniels ${ }^{1}$, H. Scott Boswell ${ }^{3}$, George E. Sandusky ${ }^{4}$, Lindsey D. Mayo $^{1}$, Reuben Kapur ${ }^{1}$, Yan Liu ${ }^{1,2}$

${ }^{1}$ Department of Pediatrics, Herman B Wells Center for Pediatric Research, Indiana University School of Medicine, Indianapolis, IN 46202; ${ }^{2}$ Department of Biochemistry and Molecular Biology, Indiana University School of Medicine, Indianapolis, IN 46202; ${ }^{3}$ Department of Medicine, Indiana University School of Medicine, Indianapolis, IN 46202; ${ }^{4}$ Department of Pathology and Laboratory Medicine, Indiana University School of Medicine, Indianapolis, IN 46202; ${ }^{5}$ Department of Rheumatism, Shanghai Municipal Hospital of Traditional Chinese Medicine, Shanghai University of Traditional Chinese Medicine, Shanghai, China.

Correspondence: Yan Liu, Ph.D., Department of Pediatrics, Herman B Wells Center for Pediatric Research, Indiana University School of Medicine, Indianapolis, IN 46202, USA. Email: liu219@iu.edu.

This is the author's manuscript of the article published in final edited form as:

Nabinger, S. C., Chen, S., Gao, R., Yao, C., Kobayashi, M., Vemula, S., ... Liu, Y. (2019). Mutant p53 enhances leukemia-initiating cell self-renewal to promote leukemia development. Leukemia, 33(6), 1535-1539. https://doi.org/10.1038/s41375-019-0377-0 
Acute myeloid leukemia (AML) is an aggressive blood cancer with poor prognosis. ${ }^{1}$ AML is thought to be initiated and maintained by a rare population of leukemia stem cells (LSCs) or leukemia-initiating cells (LICs) that have acquired the capacity for self-renewal and is blocked in their ability to differentiate by the accumulation of a series of mutations and/or epigenetic changes. ${ }^{2-3}$ Clinical studies show that LICs are resistant to conventional chemotherapy and/or targeted therapies. ${ }^{3}$ Thus, there is an unmet need to elucidate the molecular mechanisms governing LIC self-renewal and develop novel therapeutic approaches that can target LICs and improve leukemia treatment. ${ }^{3}$

The tumor suppressor p53 is a stress response protein that regulates a large number of genes in response to a variety of cellular insults, including oncogene activation, DNA damage and inflammation. ${ }^{4}$ These signals activate p53 primarily through post-translational modifications that result in augmented levels of p53 protein and transactivation activity. ${ }^{4}$ Activated p53 suppresses cellular transformation mainly by inducing growth arrest, apoptosis, DNA repair and differentiation in damaged cells. ${ }^{4}$ Accordingly, p53 function is always compromised in tumor cells, usually as a result of somatic mutations and deletions, which occur in approximately half of all human cancers. ${ }^{5}$ The TP53 gene encodes the tumor suppressor p53. The frequency of TP53 mutations in AML is approximately $10 \%$. However, in AML with complex karyotype, the frequency of TP53 mutations and/or deletions is almost $70 \% .{ }^{6}$ While TP53 mutations confer drug resistance and poor prognosis in AML, the role of mutant p53 in the initiation and progression of AML is largely unknown..$^{6-7}$

We have been investigating the role of tumor suppressor p53 in normal and malignant hematopoiesis. We found that wild type p53 maintains HSC quiescence and inhibits HSC self-renewal. ${ }^{8}$ Codon 248 of p53 is frequently mutated in $\mathrm{AML}$ and $\mathrm{p} 53^{\mathrm{R} 248 \mathrm{~W}}$ has been shown to be a gain-of function (GOF) mutant in human cancer cells as well as in animal models. ${ }^{6-7,9}$ We recently reported that $\mathrm{p} 53^{\mathrm{R} 248 \mathrm{~W}}$ enhances HSC self-renewal in steady state and promotes HSC expansion following genotoxic stresses. ${ }^{10}$ Of note, homozygous $p 53^{-/-}$and $p 53^{\mathrm{R} 248 \mathrm{~W} / \mathrm{R} 248 \mathrm{~W}}$ mice develop lymphoid tumors, including lymphoma and thymoma, but not myeloid malignancies, ${ }^{9}$ suggesting that expression of mutant p53 is not sufficient for 
inducing myeloid leukemia in mice. This has led to a search for potential second hits that cooperate with mutant p53 in the pathogenesis of myeloid malignancies, primarily focused on using mouse models.

While coexisting mutations with TP53 mutations in AML are limited, ${ }^{6-7}$ previous studies indicate that TP53 mutations co-occur with AML driver mutations in oncogenic signaling molecules such as FMSlike tyrosine kinase receptor-3 (FLT3). ${ }^{11}$ Mutations in FLT3 have been identified in myeloid malignancies, including myeloproliferative neoplasms (MPN) and AML. ${ }^{12}$ Internal tandem duplications in the juxtamembrane domain (FLT3-ITD) and mutations in the activating loop of FLT3 (FLT3-TKD) are seen in 30 to $35 \%$ of AML patients. ${ }^{12}$ Both ITD and TKD mutations of FLT3 lead to constitutive activation of the tyrosine kinase, promoting proliferation and survival of leukemic blasts. ${ }^{12}$ Given that expression of FLT3-ITD in the hematopoietic compartment results in MPN in mice and that FLT3-ITD impairs HSC self-renewal in vivo, ${ }^{13}$ we reasoned that mutant p53 might synergize with FLT3-ITD in driving the development of myeloid leukemia through enhancing LIC self-renewal.

To test this hypothesis, we generated $p 53^{R 248 W /+} F L T 3^{I T D /+}$ mice and monitored overall survival and tumor development of these mice. We observed that both $F L T 3^{I T D /+}$ and $p 53^{R 248 W /+} F L T 3^{I T D /+}$ mice have decreased life span compared to $p 53^{+/+}$mice (Figure 1a). Some $p 53^{R 248 W /+}$ mice develop myelodysplastic syndromes (MDS) with age and other $p 53^{R 248 W /+}$ mice developed lymphoma and sarcoma based upon pathological analysis of bone marrow (BM), spleen, liver, and peripheral blood (PB) (S.C. and Y.L., unpublished data). However, the majority of $p 53^{R 248 W /+} F L T 3^{I T D /+}$ mice developed MPN as seen in FLT3 ${ }^{I T D /+}$ mice (Figures $1 \mathrm{~b}, 1 \mathrm{c}$ and data not shown), suggesting that FLT3-ITD-induced MPN development does not depend on mutant p53. Histological observation of spleen sections from MPN mice showed disarray of normal splenic architecture with a reduction and almost total absence of the white pulp in some cases and increased red pulp area with increased extramedullary hematopoiesis (Figure 1c). These features appeared in conjunction with hepatosplenomegaly, variable leukocytosis and overproduction of myeloid cells in bone marrow, spleen and peripheral blood (Figure 1c). We noted that bone marrow cellularity decreased as splenomegaly increased, consistent with increased extramedullary hematopoiesis. Notably, approximately $25 \%$ of $p 53^{R 248 W /+} F L T 3^{I T D /+}$ mice developed chronic myeloid 
leukemia (CML). ${ }^{14}$ Upon necropsy, mice with CML displayed severe splenomegaly, and some also displayed hepatomegaly. Morphological analysis of peripheral blood smears revealed increased myeloid cells with dysplastic features (Figure 1c). Bone marrow cellularity varied from hypocellular to hypercellular among animals. Increased number of myeloid cells (blast to immature myeloid cells) was observed in bone marrow with extensive spread of myeloid elements in spleen and in a few livers (Figure 1c). While $p 53^{R 248 W /+} F L T 3^{I T D /+}$ mice showed marked splenomegaly compared to $p 53^{+/+}$and $F L T 3^{I T D /+}$ mice (Figure 1d), this is not likely due to CML development as majority of double-mutant mice developed MPN (Figures 1b and 1c).

Given that patients with homozygous FLT3-ITD mutations have a more severe disease compared to those with heterozygous FLT3-ITD mutations, ${ }^{11-12}$ we examined whether mutant p53 cooperates with homozygous FLT3-ITD mutant in leukemia development. We transplanted $3 \times 10^{6}$ whole bone marrow cells from $p 53^{+/+}, F L T 3^{I T D / I T D}$, or $p 53^{R 248 W /+} F L T 3^{I T D / I T D}$ mice into lethally irradiated recipient mice and measured their overall survival. Both $F L T 3^{I T D / I T D}$ and $p 53^{R 248 W /+} F L T 3^{I T D / I T D}$ recipient mice had decreased life spans compared to $p 53^{+/+}$recipient mice (Figure S1a). Interestingly, approximately $30 \%$ of the $p 53^{R 248 W /+}$ FLT3 $3^{I T D / I T D}$ transplanted animals developed CML (Figure $\mathrm{S} 1 \mathrm{~b}$ ), similar to that seen in $p 53^{R 248 W /+} F L T 3^{I T D /+}$ animals (Figure 1b). Rest of the $p 53^{R 248 W /+} F L T 3^{I T D / I T D}$ mice developed MPN (Figure S1b).

Given that some $p 53^{R 248 W /+} F L T 3^{I T D /+}$ mice develop CML, we next examined the impact of mutant p53 on FLT3-ITD ${ }^{+}$hematopoietic stem and progenitor cells (HSPCs) in order to understand the underlying mechanisms. We first analyzed peripheral blood (PB), bone marrow (BM), and spleen of $p 53^{+/+}$, $p 53^{R 248 W /+}, F L T 3^{I T D /+}$ and $p 53^{R 248 W /+} F L T 3^{I T D /+}$ mice (8 to 12 week-old). PB white blood cell (WBC) counts, BM cellularity, and spleen weight were comparable among the four groups of mice (Figures S1c, S1d, and S1e). We then examined the frequency of hematopoietic stem and progenitor cells in the BM of $p 53^{R 248 W /+} F L T 3^{I T D /+}$ mice. While the number of LT-HSCs and ST-HSCs was comparable among these mice, LSKs and MPPs were expanded in the $p 53^{R 248 W /+} F L T 3^{I T D /+}$ mice compared with that of the $p 53^{+/+}$, $p 53^{R 248 W /+}$ and $F L T 3^{I T D /+}$ mice (Figure S2a). We also observed increased frequency of myeloid progenitors 
$\left(\mathrm{Lin}^{-} \mathrm{Kit}^{+}\right.$cells) in the bone marrow of $p 53^{R 248 W /+} \mathrm{FLT3} 3^{I T D /+}$ mice (Figure S2b). These findings suggest that the effects of mutant p53 and FLT3-ITD on myeloid progenitor cell expansion appears additive. However, the number of common lymphoid progenitors (CLPs) was comparable among four group of mice (Figure $\mathrm{S} 2 \mathrm{c})$.

FLT3 mutations have been show to enhance the proliferation of hematopoietic stem and progenitor cells. ${ }^{13}$ We then examined the cell cycle status of LSKs isolated from $p 53^{+/+}, p 53^{R 248 W /+}, F L T 3^{I T D /+}$ and $p 53^{R 248 W /+} F L T 3^{I T D /+}$ mice. We confirmed that $F L T 3^{I T D /+}$ LSKs shown enhanced proliferation compared with $p 53^{+/+}$LSKs (Figure S2d). However, mutant p53 did not alter the proliferation of FLT3 $3^{I T D /+}$ LSKs (Figure S2d). To determine the impact of mutant p53 on myeloid progenitors, we performed serial replating assays using $\mathrm{BM}$ cells from $p 53^{+/+}, p 53^{R 248 W /+}, F L T 3^{I T D /+}$ and $p 53^{R 248 W /+} F L T 3^{I T D /+}$ mice. While the colony formation potential of $p 53^{+/+}$and $F L T 3^{I T D /+}$ BM cells was comparable in serial replating assays, $p 53^{R 248 W /+} F L T 3^{I T D /+}$ BM cells show enhanced replating potential compared to $p 53^{R 248 W /+}$ and $F L T 3^{I T D /+}$ BM cells (Figure 2a), suggesting that expanded myeloid progenitors in $p 53^{R 248 W /+} F L T 3^{I T D /+}$ mice are functional in vitro.

To examine the impact of mutant p53 on HSCs in vivo, we performed serial competitive bone marrow transplantation assays. We transplanted $5 \times 10^{5}$ donor BM cells $\left(p 53^{+/+}, p 53^{R 248 W /+}, F L T 3^{I T D /+}\right.$ or $\left.p 53^{R 248 W /+} F L T 3^{I T D /+}, \mathrm{CD} 45.2^{+}\right)$into lethally irradiated $(11 \mathrm{~Gy}) \mathrm{F} 1$ recipient mice $\left(\mathrm{CD} 45.1^{+} \mathrm{CD} 45.2^{+}\right)$along with $5 \times 10^{5}$ competitor BM cells $\left(\mathrm{CD} 45.1^{+}\right)$. Peripheral blood white blood cell counts were comparable among the four groups of mice following transplantation (Figure S2e). While $F L T 3^{I T D /+}$ BM cells showed decreased repopulating ability compared to $p 53^{+/+}$cells 16 weeks post transplantation, $p 53^{R 248 W /+} F L T 3^{I T D /+}$ BM cells displayed enhanced engraftment compared to $F L T 3^{I T D /+}$ BM cells (Figure 2b). We then sacrificed the recipient mice and examined the frequency of donor-derived hematopoietic stem and progenitor cells in their bone marrow. We found increased number of donor-derived LSKs in the $\mathrm{BM}$ of recipient mice repopulated with $p 53^{R 248 W /+} \mathrm{BM}$ cells compared to that of the $p 53^{+/+}$and $F L T 3^{I T D /+} \mathrm{BM}$ cells, whereas the frequency of donor-derived LSKs in the BM of recipient mice repopulated with $p 53^{R 248 W /+}$ and $p 53^{R 248 W /+} F L T 3^{I T D /+}$ cells was comparable (Figure S2f). We found 
increased number of donor-derived GMPs in the BM of recipient mice repopulated with $p 53^{R 248 W /+} F L T 3^{I T D /+}$ bone marrow cells compared to that of the FLT3 ${ }^{I T D /+}$ BM cells (Figure S3a). The spleen size was comparable in recipient mice repopulated with four group of BM cells (Figure S3b).

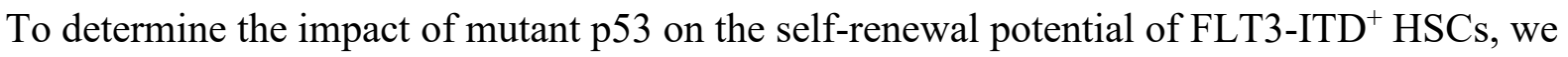
transplanted $3 \times 10^{6} \mathrm{BM}$ cells isolated from the primary recipient mice repopulated with $p 53^{+/+}, p 53^{R 248 W /+}$, $F L T 3^{I T D /+}$ or $p 53^{R 248 W /+} F L T 3^{I T D /+}$ cells into lethally irradiated secondary F1 recipients. Sixteen weeks after transplantation, $p 53^{R 248 W /+} F L T 3^{I T D /+}$ cells continued to show increased repopulating ability compared to $F L T 3^{I T D /+} \mathrm{BM}$ cells (Figure 2c). These findings suggest that mutant p53 may promote leukemic transformation through enhancing LIC self-renewal.

To examine the impact of mutant p53 on oncogenic signaling pathways, we performed western blot analysis on macrophage progenitor cells derived from $p 53^{+/+}, p 53^{R 248 W /+}, F L T 3^{I T D /+}$ or $p 53^{R 248 W /+} F L T 3^{I T D /+}$ bone marrow cells. Consistent with previous studies, cells from $F L T 3^{I T D /+}$ mice had activated FAK, STAT5, and AKT (Figure 2d). Further, expressing FLT3-ITD in a mutant p53 background enhances activated ERK levels but slightly decreases activated FAK and STAT5 levels (Figure 2d). We found increased levels of FLT3 in $p 53^{R 248 W / 4} F L T 3^{I T D /+}$ macrophage progenitor cells (Figure S3c). Thus, expressing FLT3-ITD in a mutant p53 background has no effect on FLT3-ITD-induced activation of signaling pathways. However, ERK inhibitor treatment decreased the replating potential of $p 53^{R 248 W /+}$, $F L T 3^{I T D /+}$ and $p 53^{R 248 W /+} F L T 3^{I T D /+}$ bone marrow cells (Figure S3d). These findings suggest that mutant p53 and FLT3-ITD may function through different signaling pathways in the pathogenesis of hematological malignancies. In the future, we will elucidate the mechanisms by which mutant p53 upregulates FLT3 in HSPCs.

While TP53 and FLT3 mutations are rarely co-occur in MPN and AML, ${ }^{11}$ the underlying mechanisms are not known. We found that the majority of $p 53^{R 248 W /+} F L T 3^{I T D /+}$ mice developed MPN, as seen in $F L T 3^{I T D /+}$ mice. ${ }^{13}$ Further, we discovered that mutant p53 and FLT3-ITD cooperate in CML development in mice. Functionally, mutant p53 synergizes with FLT3-ITD to expand the myeloid progenitor cell pool and enhance the self-renewal potential of LICs. TP53 mutations are present in both 
chronic and blast crisis phase of CML, ${ }^{15}$ underscoring the importance of mutant $\mathrm{p} 53$ in CML pathogenesis. Delineating the role of mutant p53 and FLT3-ITD in LIC self-renewal and pathogenesis of hematological malignancies may facilitate the development of novel therapeutic approaches that can improve leukemia treatment.

\section{CONFLICT OF INTEREST}

The authors declared that no conflicts of interest exists.

\section{ACKNOWLEDGEMENTS}

This work was supported by the Office of the Assistant Secretary of Defense for Health Affairs, through the Bone Marrow Failure Research Program - Idea Development Award under Award No. W81XWH-181-0265 to YL. Opinions, interpretations, conclusions and recommendations are those of the author and are not necessarily endorsed by the Department of Defense. This work was also supported in part by two NIH R56 Awards (R56DK119524-01 and R56AG05250), a DoD Career Development Award W81XWH13-1-0187, a Scholar Award from the St. Baldrick's Foundation, an Elsa Pardee Foundation New Investigator Award, a Leukemia Research Foundation New Investigator Award, a Showalter Trust Fund New Investigator Award, an Alex Lemonade Stand Foundation grant, a Children's Leukemia Research Association grant, and an American Cancer Society Institutional Research Grant to YL. SCN was supported by a NIH F32 Award 1F32CA203049-01. The authors would like to acknowledge the Flow Cytometry Core and In vivo Therapeutic Core Laboratories, which were sponsored, in part, by the NIDDK Cooperative Center of Excellence in Hematology (CCEH) grant U54 DK106846. This work was supported, in part, by a Project Development Team within the ICTSI NIH/NCRR Grant Number UL1TR001108. We would like to thank Dr. Yang Xu at USCD for providing the $\mathrm{p} 53^{\mathrm{R} 248 \mathrm{~W}}$ mice to the study. 


\section{AUTHOR CONTRIBUTIONS}

SCN and YL Designed the research. SCN, SC, RG, CY, MK, SV, ACF, CW, and CD Performed the research. SCN, SC, and YL Analyzed the data and performed the statistical analysis. GES Performed pathological analysis. HSB, LDM, and RK Provided reagents and constructive advice to the study. SCN, SC, and YL Wrote the manuscript. All authors read, commented on, and approved the manuscript. 


\section{REFERENCES}

1. Coombs CC, Tallman MS, Levine RL. Molecular therapy for acute myeloid leukaemia. Nat Rev Clin Oncol 2016;13:305-18.

2. Hope KJ, Jin L, Dick JE. Acute myeloid leukemia originates from a hierarchy of leukemic stem cell classes that differ in self-renewal capacity. Nat Immunol 2004; 5:738-43.

3. Essers MA, Trumpp A. Targeting leukemic stem cells by breaking their dormancy. Mol Oncol 2010; 4: 443-50.

4. Khoo KH, Verma CS, Lane DP. Drugging the p53 pathway: understanding the route to clinical efficacy. Nat Rev Drug Discov 2014; 13:217-36.

5. Brosh R, Rotter V. When mutants gain new powers: news from the mutant p53 field. Nat Rev Cancer 2009; 9:701-13.

6. Rücker FG, Schlenk RF, Bullinger L, Kayser S, Teleanu V, Kett H et al. TP53 alterations in acute myeloid leukemia with complex karyotype correlate with specific copy number alterations, monosomal karyotype, and dismal outcome. Blood 2012; 119: 2114-21.

7. Prokocimer M, Molchadsky A, Rotter V. Dysfunctional diversity of p53 proteins in adult acute myeloid leukemia: projections on diagnostic workup and therapy. Blood 2017;130:699-712.

8. Liu Y, Elf SE, Miyata Y, Sashida G, Liu YH, Huang G et al. p53 Regulates Hematopoietic Stem Cell Quiescence. Cell Stem Cell 2009; 4: 37-48.

9. Song H, Hollstein M, Xu Y. p53 gain-of-function cancer mutants induce genetic instability by inactivating ATM. Nat Cell Biol 2007; 15: 376-88.

10. Chen S, Gao R, Yao C, Kobayashi M, Liu SZ et al. Genotoxic stresses promotes the clonal expansion of hematopoietic stem cells expressing mutant p53. Leukemia 2018; 32:850-854.

11. Garg M, Nagata Y, Kanojia D, Mayakonda A, Yoshida K, Haridas Keloth S et al. Profiling of somatic mutations in acute myeloid leukemia with FLT3-ITD at diagnosis and relapse. Blood 2015;126:2491-501. 
12. Swords R, Freeman C, Giles F. Targeting the FMS-like tyrosine kinase 3 in acute myeloid leukemia. Leukemia 2012; 26:2176-85.

13. Lee BH, Tothova Z, Levine RL, Anderson K, Buza-Vidas N et al. FLT3 mutations confer enhanced proliferation and survival properties to multipotent progenitors in a murine model of chronic myelomonocytic leukemia. Cancer Cell 2007;12:367-80.

14. Morse HC 3rd, Anver MR, Fredrickson TN, Haines DC, Harris AW, Harris NL, Jaffe ES, Kogan SC, MacLennan IC, Pattengale PK, Ward JM; Hematopathology subcommittee of the Mouse Models of Human Cancers Consortium. Bethesda proposals for classification of lymphoid neoplasms in mice. Blood 2002;100:246-58.

15. Menezes J, Salgado RN, Acquadro F, Gómez-López G, Carralero MC, Barroso A, Mercadillo F, Espinosa-Hevia L, Talavera-Casañas JG, Pisano DG, Alvarez S, Cigudosa JC. ASXL1, TP53 and IKZF3 mutations are present in the chronic phase and blast crisis of chronic myeloid leukemia. Blood Cancer J 2013;3:e157. 


\section{FIGURE LEGENDS}

Figure 1. Mutant p53 cooperates with FLT3-ITD in the pathogenesis of myeloid leukemia. (a) FLT3 $3^{I T D /+}$ and $p 53^{R 248 W /+} F L T 3^{I T D /+}$ mice show decreased survival compared to $p 53^{+/+}$mice $\left(\mathrm{n}=11, p 53^{+/+} ; \mathrm{n}=11\right.$, $\left.F L T 3^{I T D /+} ; \mathrm{n}=12, p 53^{R 248 W /+} F L T 3^{I T D /+}, * * \mathrm{p}<0.01, * * * * \mathrm{p}<0.0001\right)$. (b) Disease spectrums in $F L T 3^{I T D /+}$ and $p 53^{R 248 W /+} F L T 3^{I T D /+}$ mice were determined by pathological analysis of bone marrow, spleen, liver, and peripheral blood (n=9, FLT3 $\left.3^{I T D /+} ; \mathrm{n}=12, p 53^{R 248 W /+} F L T 3^{I T D /+}\right)$. (c) Representative H\&E (20X) images of bone marrow, spleen, liver and peripheral blood smears from $F L T 3^{I T D /+}$ mice with MPN and $p 53^{R 248 W /+} F L T 3^{I T D /+}$ mice with CML. (d) Spleen weight of $p 53^{+/+}, F L T 3^{I T D /+}$, and $p 53^{R 248 W /+} F L T 3^{I T D /+}$ mice. Mean values $\left( \pm\right.$ SEM) are shown $\left(\mathrm{n}=12, p 53^{+/+} ; \mathrm{n}=12, F L T 3^{I T D /+} ; \mathrm{n}=17, p 53^{R 248 W /+} F L T 3^{I T D /+}\right.$, $* \mathrm{p}<0.05)$.

Figure 2. Mutant p53 enhances the self-renewal potential of FLT3-ITD ${ }^{+}$LICs. (a) Serial replating assays of bone marrow cells from young $p 53^{+/+}, p 53^{R 248 W /+}, F L T 3^{I T D /+}$ and $p 53^{R 248 W /+} F L T 3^{I T D /+}$ mice. Mean values $( \pm \mathrm{SD})$ are shown $\left(\mathrm{n}=3,{ }^{*} \mathrm{p}<0.05, * * * * \mathrm{p}<0.0001\right)$. (b) $\mathrm{p} 53^{\mathrm{R} 248 \mathrm{~W}}$ enhances the repopulating potential of $F L T 3^{I T D /+}$ hematopoietic cells. Percentage of donor-derived (CD45.2 ${ }^{+}$) cells in the peripheral blood of primary recipient mice post-transplantation, measured at 4-week intervals. Mean values $( \pm \mathrm{SEM})$ are shown $\left(\mathrm{n}=7,{ }^{* * *} \mathrm{p}<0.001\right)$. (c) The percentage of donor-derived cells in the peripheral blood of secondary recipient mice. Mean values $( \pm \mathrm{SEM})$ shown, $\left(\mathrm{n}=7, p 53^{\mathrm{R} 248 \mathrm{~W} /+}\right.$ vs $F L T 3^{I T D /+}$ and $F L T 3^{I T D /+}$ vs $p 53^{R 248 W /+} F L T 3^{I T D /+}, * * * * \mathrm{p}<0.0001$ ). (d) Western blot analysis of activated and total FAK, STAT5, AKT, and ERK protein levels in $p 53^{+/+}, p 53^{R 248 W /+}, F L T 3^{I T D /+}$ and $p 53^{R 248 W /+} F L T 3^{I T D /+}$ mononuclear cells differentiated into macrophage progenitors. Loading controls GAPDH and Vinculin are also shown. Quantification of phosphorylated proteins was calculated relative to total protein level and is displayed above each respective phospho-protein. 
a

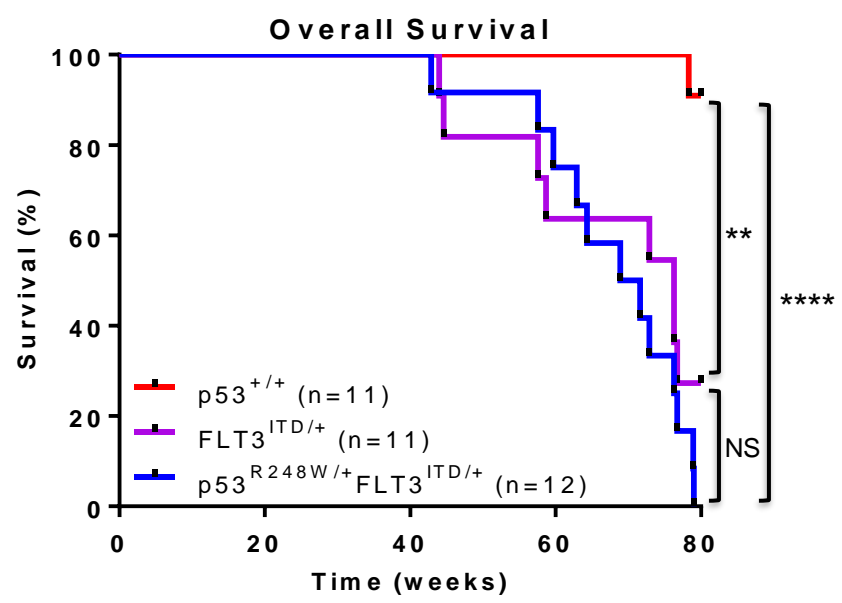

b

\begin{tabular}{|c|c|c|}
\hline Disease Type & $\begin{array}{c}\mathrm{FLT3}^{\mathrm{ITD} /+} \\
(\mathrm{n}=9)\end{array}$ & $\begin{array}{c}\mathrm{p5}^{\mathrm{R} 248 \mathrm{~W} /+} \mathrm{FLT3}^{\mathrm{ITD} /+} \\
(\mathrm{n}=12)\end{array}$ \\
\hline MPN & $9 / 9(100 \%)$ & $9 / 12(75 \%)$ \\
\hline $\mathrm{CML}$ & 0 & $3 / 12(25 \%)$ \\
\hline
\end{tabular}

d
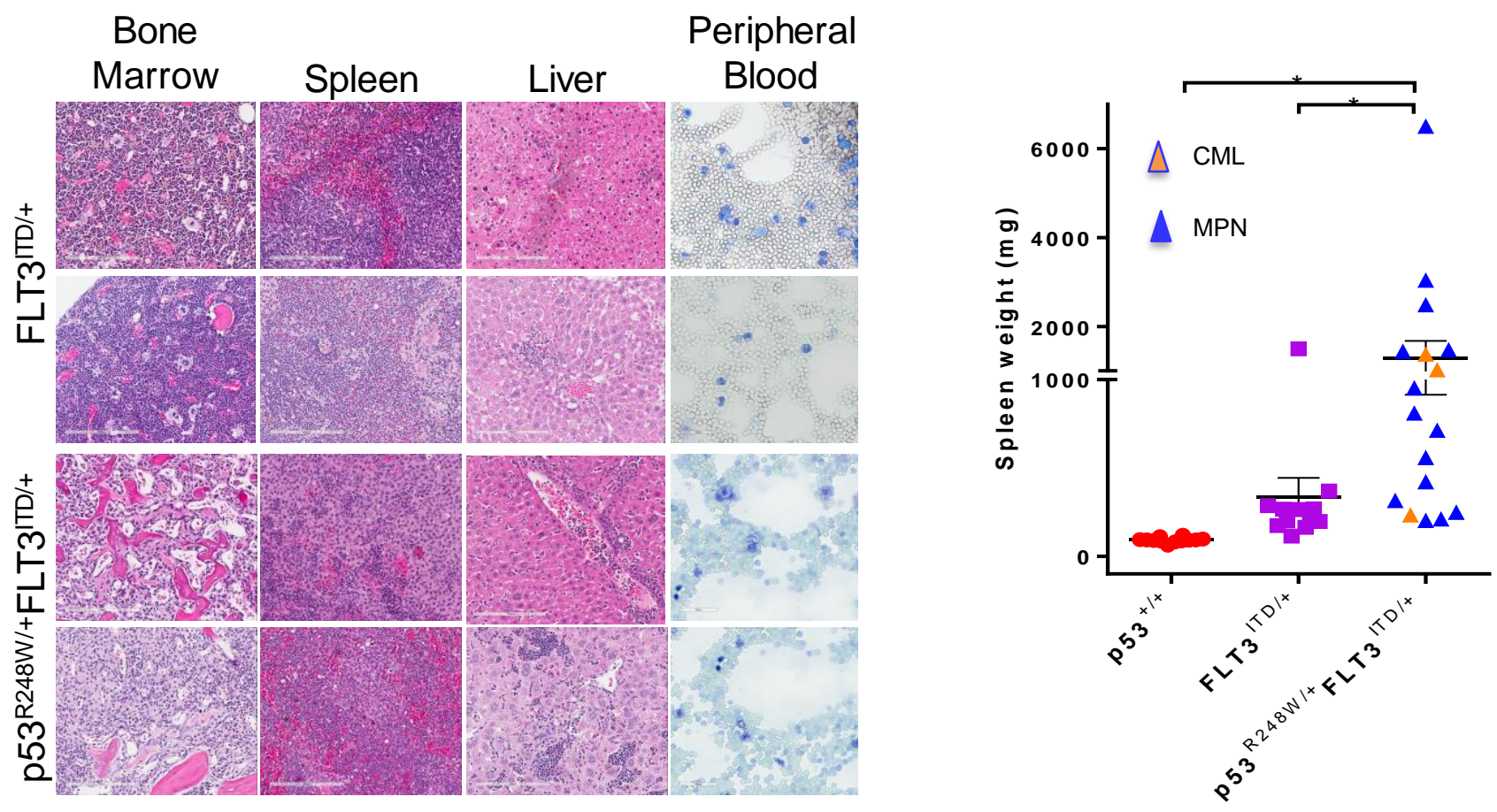
a

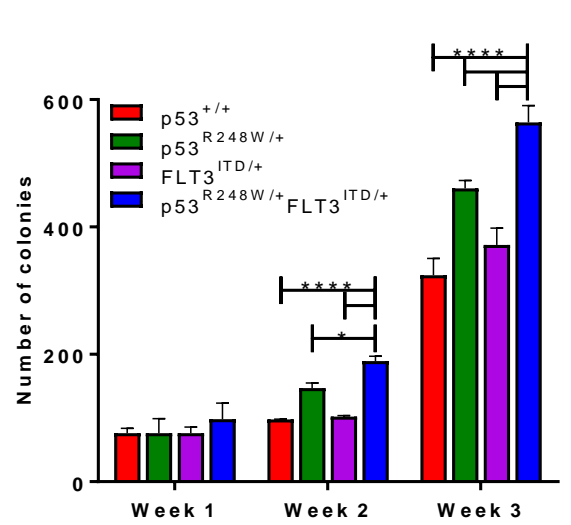

b

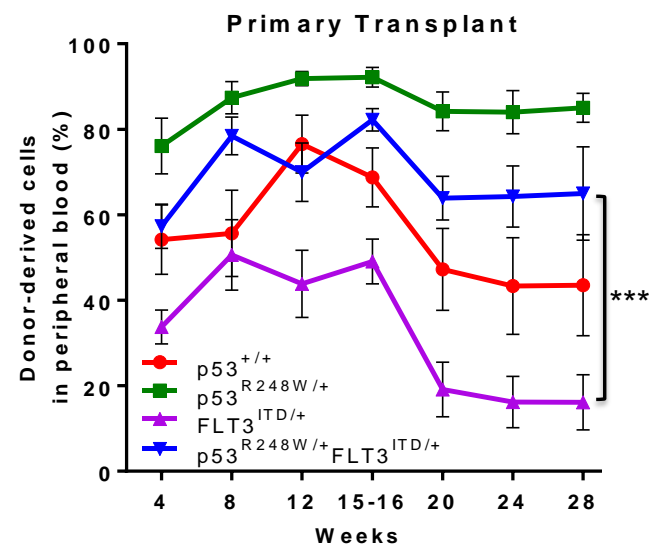

C

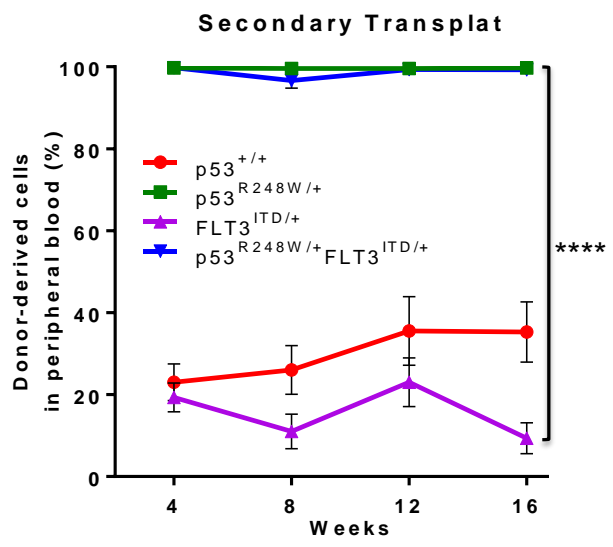

d
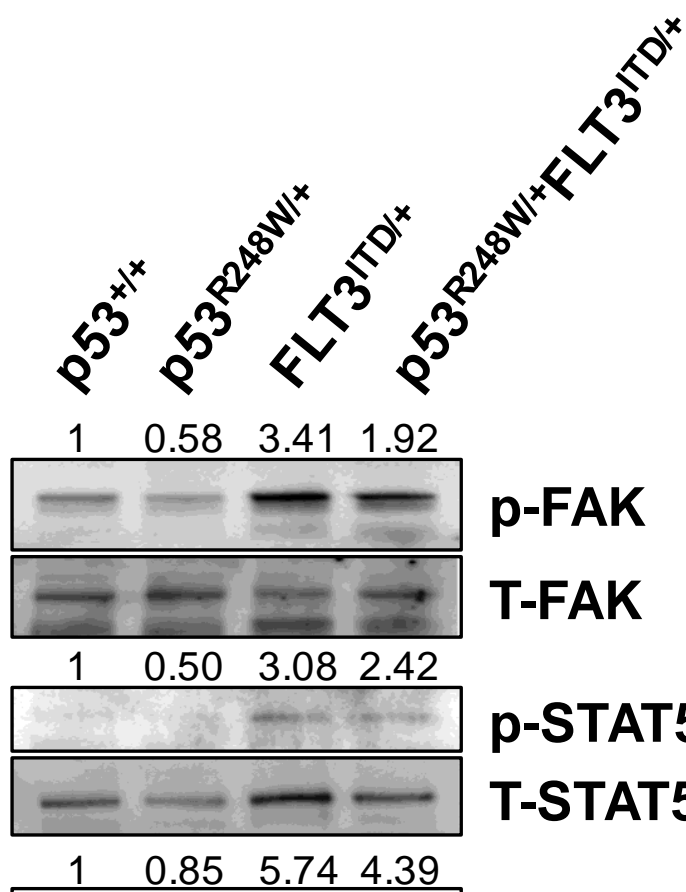

p-FAK

T-FAK

p-STAT5

T-STAT5

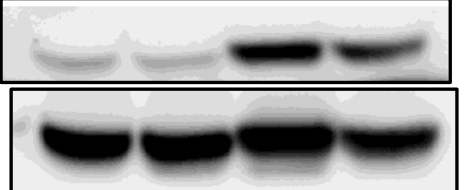

p-AKT

$\begin{array}{llll}1 & 1.13 & 2.38 & 5.47\end{array}$

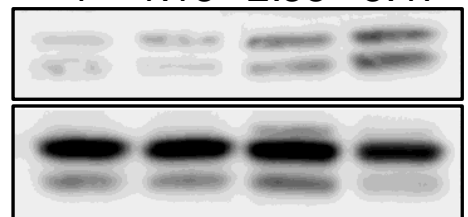

p-ERK

T-ERK

T-AKT

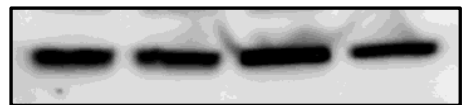

GAPDH

Vinculin 


\section{Mutant p53 enhances leukemia-initiating cell self-renewal to promote leukemia development}

\section{Supplemental Methods}

\section{Mice}

FLT3-ITD knock-in mice were obtained from the Jackson Laboratory (Lee et al., 2007). The humanized mutant $p 53$ knock-in mice ( $p 53^{\mathrm{R} 248 \mathrm{~W} /+}$ ) have been backcrossed to the $C 57 B L 6$ background for at least 8 generations (Chen et al., 2018). Wild type C57BL/6 (CD45.2 $)$, B6.SJL (CD45.1 $\left.{ }^{+}\right)$and F1 mice (CD45.2 ${ }^{+}$ CD $45.1^{+}$) mice were obtained from an on-site core breeding colony. All mice were maintained in the Indiana University Animal Facility according to IACUC-approved protocols.

\section{Flow Cytometry}

Flow cytometry analysis of hematopoietic stem and progenitor cells was performed as described previously (Liu et al., 2009). Murine hematopoietic stem and progenitor cells were identified and evaluated by flow cytometry using a single cell suspension of bone marrow mononuclear cells (BMMCs). Hematopoietic stem and progenitors are purified based upon the expression of surface markers. Bone marrow (BM) cells were obtained from femurs by flushing cells out of the bone using a syringe and phosphate-buffered saline (PBS) with 2mM EDTA. Red blood cells (RBCs) were lysed by RBC lysis buffer (eBioscience) prior to staining. Experiments were performed on FACS LSR IV cytometers (BD Biosciences) and analyzed by using the FlowJo Version 9.3.3 software (TreeStar).

\section{Serial Replating Assays}

Clonogenic progenitors were determined in methylcellulose medium (MethoCult GF M3434, StemCell Technologies) with cytokines (SCF, TPO, EPO, IL-3 and GM-CSF) using 2 x 10 4 BMMCs per well (6well plate). Colonies were scored after 7 days of the initial culture, and all cells were collected and washed twice in phosphate-buffered saline. Subsequently cells were cultured at $2 \times 10^{4}$ per well in the same medium. Colony scoring and replating were repeated every 7 days for at least two times, or until no colonies were observed in the cultures. 


\section{Transplantation}

For the competitive repopulation assays, we injected $5 \times 10^{5} \mathrm{BM}$ cells from $p 53^{+/+}, p 53^{R 248 W /+}, F L T 3^{I T D /+}$ and $p 53^{R 248 W /+} F L T 3^{I T D /+}$ mice $\left(\mathrm{CD} 45.2^{+}\right)$plus $5 \times 10^{5}$ competitor BM cells $\left(\mathrm{CD} 45.1^{+}\right)$into lethally irradiated (11Gy) F1 mice (CD45.1 $\left.{ }^{+} \mathrm{CD} 45.2^{+}\right)$. Peripheral blood was obtained by tail vein bleeding every 4-week after transplantation, RBC lysed, and the PB mononuclear cells stained with anti-CD45.2 FITC and anti-CD45.1 PE, and analyzed by flow cytometry. 16 weeks following transplantation, bone marrow cells from recipient mice were analyzed to evaluate donor chimerism in bone marrows. For secondary transplantation, $3 \times 10^{6} \mathrm{BM}$ cells from mice reconstituted with $p 53^{+/+}, p 53^{R 248 W /+}, F L T 3^{I T D /+}$ and $p 53^{R 248 W /+} F L T 3^{I T D /+}$ BM cells were injected into lethally irradiated $\mathrm{F} 1$ mice $\left(\mathrm{CD} 45.1^{+} \mathrm{CD} 45.2^{+}\right)$.

\section{Western Blot Analysis}

Bone marrow mononuclear cells from $p 53^{+/+}, p 53^{R 248 W /+}, F L T 3^{I T D /+}$ and $p 53^{R 248 W /+} F L T 3^{I T D /+}$ mice were differentiated into macrophage progenitors with 10ng/ml M-CSF for 7-10 days. Total cell lysates were collected, run on SDS-PAGE gels, and transferred to PVDF membrane. Blots were probed with indicated primary antibody [p-FAK(Cell Sig. \#3283), T-FAK(BD \#610087), p-STAT5(Cell Sig. \#9351), TSTAT5(Cell Sig. \#9363), p-AKT(Cell Sig. \#9271), T-AKT(Cell Sig. \#4691 ), p-ERK(Cell Sig. \#9101), T-ERK(Cell Sig. \#9102), FLT3 (Cell Signaling. \#3462S), GAPDH(Santa Cruz \#sc-32233), or Vinculin(sc-73614)] and incubated with secondary antibody, and imaged.

\section{Tissue Specimens}

All mice tissue samples were collected following a detailed LARC approved lab animal protocol, and were approved by the Indiana University School of Medicine Institutional Animal Care and Use Committee and adhered to all standards set forth in the ARVO Statement for the Use of Animals in Research.

\section{Tissue Processing}


In the histology study, all mice tissues were fixed in $10 \%$ neutral buffered formalin at $4^{\circ} \mathrm{C}$ for 24 hours following tissue processing, and then embedded in paraffin. Five-micrometer sections were stained for routine $\mathrm{H} \& \mathrm{E}$.

\section{Pathology Scoring}

The lymphomas were sub classified into the following groups based on the St. Jude's pathology classification of mouse lymphomas (Ward JM et al., 2012; Frith CH et al., 1993): 1: Diffuse Large B Cell Lymphoma involving multiple organs, 2: Thymic Lymphoma (usually associated with thymic and around the heart), and 3: Follicular Lymphoma, seen as a diffuse proliferation of lymphocytes in the white pulp area in the spleen.

Myeloid proliferations include a wide range of disease, those similar to human disease at one end to subtle increases in the splenic nonlymphoid hematopoietic cells in genetic engineered mice at the other end. These can progress to leukemias. The mouse myeloproliferative disease is broken down into two subclassisfication, one in genetic engineered mice and in naturally occurring mice. This is characterized by the combination of the following: A. Erythrocytosis, leukocytosis of myeloid cells, and/or thrombocytosis/circulating micromegakaryocytes; B. Increased in non-lymphoid hematopoietic cells in the spleen and bone marrow. Chronic myeloid leukemia (CML) in the mouse is a progression of myeloproliferative disease and is characterized by at least $20 \%$ immature blast cells with moderate differentiation and neutrophilic in blood, spleen, and or bone marrow (Kogan et al., Blood, 2002).

The leukemia were classified by the following criteria: 1 . Presence of large lymphocytes in the blood vessel lumens and/or vascular channels in the following organs examined. 2. Liver, lung, and kidney (blood vessels) and 3. Bone marrow and spleen (vascular channels). 
The MPN were classified as follows: 1) myeloid proliferation in the bone marrow, spleen and liver. 2) Myelofibrosis in the bone marrow. This was composed of a proliferative infiltrate of mononuclear cells with features of blast and immature myeloid cells in the bone marrow, liver, and spleen.

All histology slides were hand read by a pathologist and the cause of death was determined. All images obtained were scanned using Aperio Whole Slide Digital Imaging Platform.

\section{Statistical Analysis}

Statistical analysis was performed with GraphPad Prism 6 software (GraphPad software, Inc). All data are presented as mean \pm standard error of the mean (SEM). The sample size for each experiment are included in the figure legends. Statistical analyses were performed using unpaired, two-tailed Student's t test where applicable for comparison between two groups, and a One-way ANOVA test or Two-way ANOVA was used for experiments involving more than two groups. Survival curves were tested using Geham-Breslow-Wilcoxon test and those P-values were then tested using 'analyze stack of P values' to check significance. Statistical significance was defined as $* \mathrm{p}<0.05 ; * * \mathrm{p}<0.01 ; * * * \mathrm{p}<0.001 ; * * * \mathrm{p}<$ 0.0001; ns, not significant.

\section{Supplementary Figure Legends}

\section{Figure S1.}

(a) Recipient mice repopulated with $F L T 3^{I T D / I T D}$ and $p 53^{R 248 W /+} F L T 3^{I T D / I T D}$ bone marrow cells show significantly reduced survival compared to that of the $p 53^{+/+}$cells $\left(\mathrm{n}=6, p 53^{+/+} ; \mathrm{n}=4\right.$, FLT $3^{\text {ITD/ITD }}$; and $\mathrm{n}=6$, $p 53^{R 248 W /+} F L T 3^{I T D / I T D}$ mice, **p<0.01). (b) Disease spectrums in $F L T 3^{I T D / I T D}$ and $p 53^{R 248 W /+} F L T 3^{I T D / I T D}$ whole bone marrow transplant recipients were determined by pathological analysis of bone marrow, spleen, liver, and peripheral blood $\left(\mathrm{n}=4, F L T 3^{I T D / I T D} ; \mathrm{n}=6, p 53^{R 248 W /+} F L T 3^{I T D / I T D}\right.$ mice). (c) Peripheral white blood cell $(\mathrm{WBC})$ counts in $p 53^{+/+}, p 53^{R 248 W /+}, F L T 3^{I T D /+}$ and $p 53^{R 248 W /+} F L T 3^{I T D /+}$ mice. Mean values $\left( \pm\right.$ SEM) are shown $(\mathrm{n}=6)$. (d) Bone marrow cell numbers of young $p 53^{+/+}, p 53^{R 248 W /+}, F L T 3^{I T D /+}$ 
and $p 53^{R 248 W /+} F L T 3^{I T D /+}$ mice. Mean values $( \pm \mathrm{SEM})$ are shown $(\mathrm{n}=6)$. (e) Spleen weight of young $p 53^{+/+}$, $p 53^{R 248 W /+}, F L T 3^{I T D /+}$ and $p 53^{R 248 W /+} F L T 3^{I T D /+}$ mice. Mean values $( \pm \mathrm{SEM})$ are shown $(\mathrm{n}=8)$.

Figure S2. (a) Frequency of LT-HSCs (CD48-CD150+LSKs), ST-HSCs (CD48-CD150-LSKs), MPPs $\left(\mathrm{CD} 48^{+} \mathrm{CD} 150-\mathrm{LSKs}\right)$ and LSKs in the $\mathrm{BM}$ of young $p 53^{+/+}, p 53^{R 248 W /+}, F L T 3^{I T D /+}$ and $p 53^{R 248 W /+} F L T 3^{I T D /+}$ mice. Mean values $( \pm$ SEM) are shown $(\mathrm{n}=8, * \mathrm{p}<0.05, * * \mathrm{p}<0.01, * * * \mathrm{p}<0.001) .(\mathbf{b})$ The frequency of myeloid progenitor cells $\left(\mathrm{Lin}^{-} \mathrm{Kit}^{+}\right), \mathrm{CMPs}\left(\mathrm{Lin}^{-} \mathrm{Sca}^{-} \mathrm{Kit}^{+} \mathrm{Fc} \gamma \mathrm{RII} / \mathrm{III}^{\mathrm{low}} \mathrm{CD} 34^{\text {high }}\right), \mathrm{GMPs}$ $\left(\mathrm{Lin}^{-} \mathrm{Sca} 1^{-} \mathrm{Kit}^{+} \mathrm{Fc} \gamma \mathrm{RII} / \mathrm{III} \mathrm{I}^{\text {high }} \mathrm{CD} 34^{\text {high }}\right)$, and MEPs $\left(\mathrm{Lin}^{-} \mathrm{Sca}^{-} \mathrm{Kit}^{+} \mathrm{Fc} \gamma \mathrm{RII} / \mathrm{III}^{\text {low }}{ }^{\mathrm{CD}} 34^{\text {low }}\right)$ in the bone marrow of young $p 53^{+/+}, p 53^{R 248 W /+}, F L T 3^{I T D /+}$ and $p 53^{R 248 W /+} F L T 3^{I T D /+}$ mice. Mean values $( \pm$ SEM) are shown $(\mathrm{n}=8, * \mathrm{p}<0.05, * * \mathrm{p}<0.01, * * * * \mathrm{p}<0.0001$. (c) The frequency of common lymphoid progenitors (CLPs) in the bone marrow of young $p 53^{+/+}, p 53^{R 248 W /+}, F L T 3^{I T D /+}$ and $p 53^{R 248 W /+} F L T 3^{I T D /+}$ mice. Mean values $\left( \pm\right.$ SEM) are shown $(\mathrm{n}=5)$. (d) Cell cycle status of LSK cells from young $p 53^{+/+}, p 53^{R 248 W /+}$, $F L T 3^{I T D /+}$ and $p 53^{R 248 W /+} F L T 3^{I T D /+}$ mice was determined by flow cytometry analysis. Mean values $( \pm$ SEM) are shown $(n=4, * p<0.05)$. (e) Peripheral white blood cell counts in recipient mice at 16 weeks following bone marrow transplantation. Mean values $( \pm S E M)$ shown $(n=7)$. (f) The frequency of donorderived LSKs, LT-HSCs, ST-HSCs, and MPPs in the bone marrow of primary recipient mice 16 weeks following transplantation. Mean values $( \pm \operatorname{SEM})$ shown $(n=7, * p<0.05, * * * p<0.001)$.

Figure S3. (a) The frequency of donor-derived GMPs, CMPs, and MEPs in the bone marrow of primary recipient mice 16 weeks following transplantation. Mean values $( \pm$ SEM) shown $(n=7, * * p<0.01)$. (b) Spleen weight of recipient mice at 16 weeks following bone marrow transplantation. Mean values $( \pm$ SEM) shown $(\mathrm{n}=7)$. (c) Western blot analysis of FLT3 in $p 53^{+/+}, p 53^{R 248 W /+}, F L T 3^{I T D /+}$ and $p 53^{R 248 W /+} F L T 3^{I T D /+}$ mononuclear cells differentiated into macrophage progenitors. Loading controls GAPDH and Vinculin are also shown. (d) ERK inhibitor treatment decreased the replating potential of $p 53^{R 248 W /+}, F L T 3^{I T D /+}$ and $p 53^{R 248 W /+} F L T 3^{I T D /+}$ bone marrow cells. Mean values $( \pm \mathrm{SD})$ are shown $\left(\mathrm{n}=3,{ }^{*} \mathrm{p}<0.05, * * \mathrm{p}<0.01\right)$. 


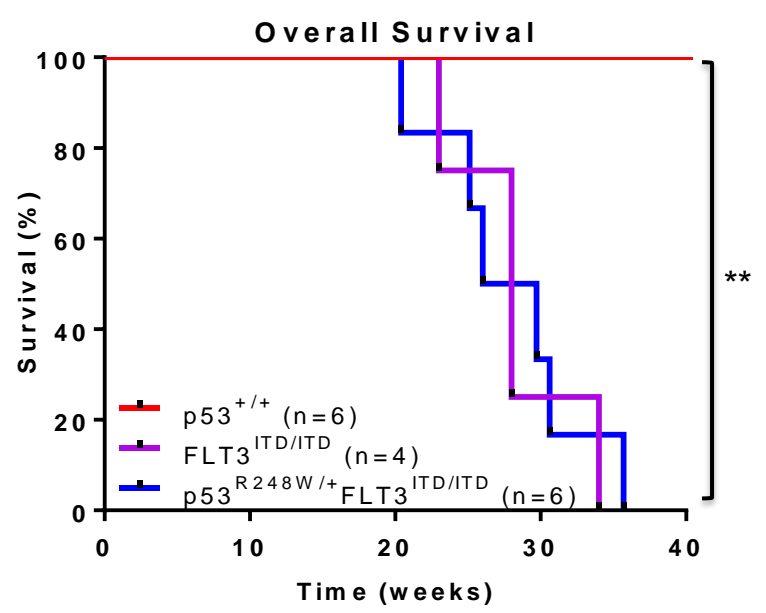

\begin{tabular}{|c|c|c|}
\hline Disease Type & $\begin{array}{c}\text { FLT3 }^{\text {ITD/TD }} \\
(n=4)\end{array}$ & $p^{\text {p53248W/+ FLT3 }} \begin{array}{c}\text { ITD/TD } \\
(n=6)\end{array}$ \\
\hline $\begin{array}{c}\text { MPN and } \\
\text { Lymphoma }\end{array}$ & $4 / 4(100 \%)$ & $4 / 6(67 \%)$ \\
\hline $\begin{array}{c}\text { CML and } \\
\text { Lymphoma }\end{array}$ & 0 & $2 / 6(33 \%)$ \\
\hline
\end{tabular}
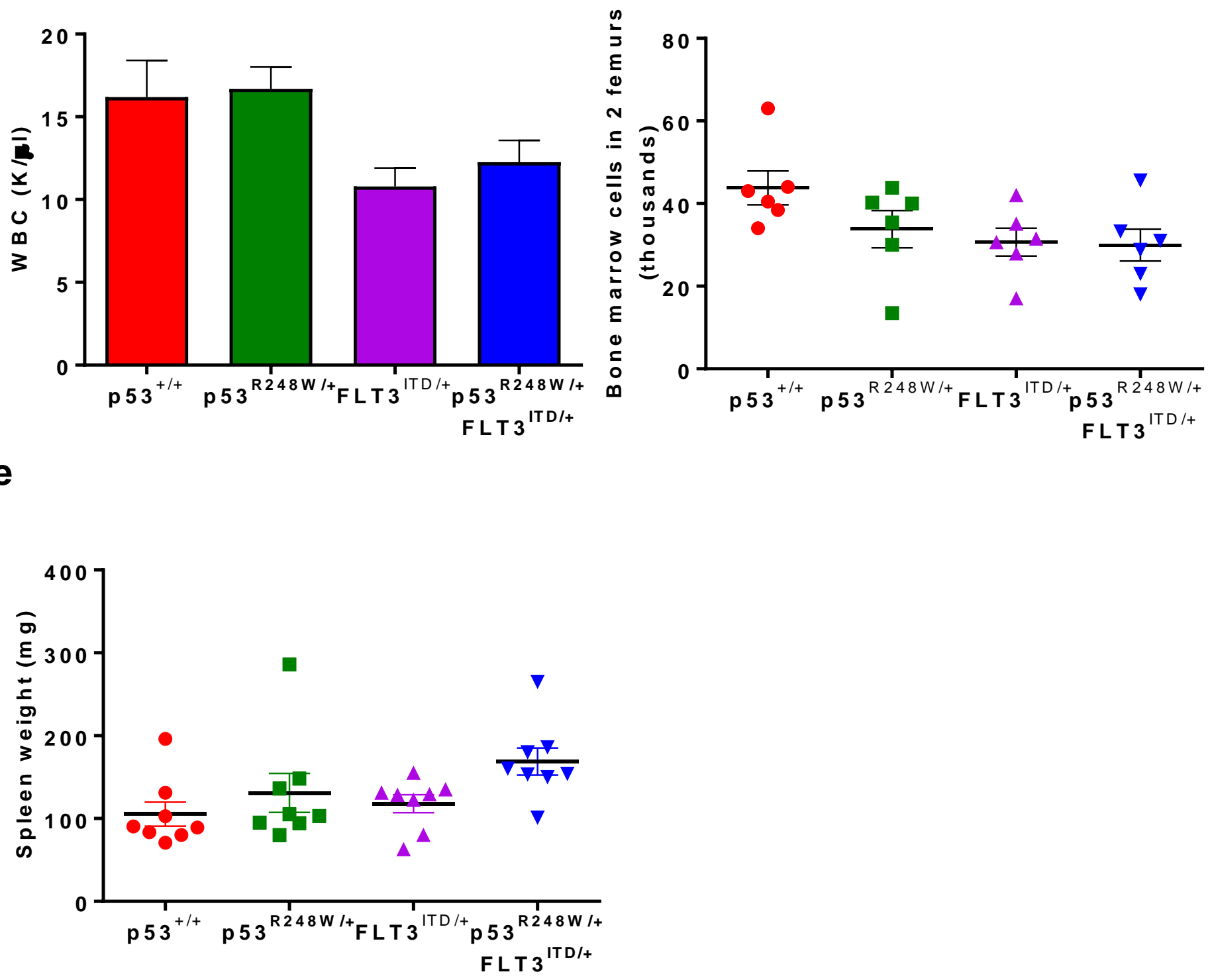

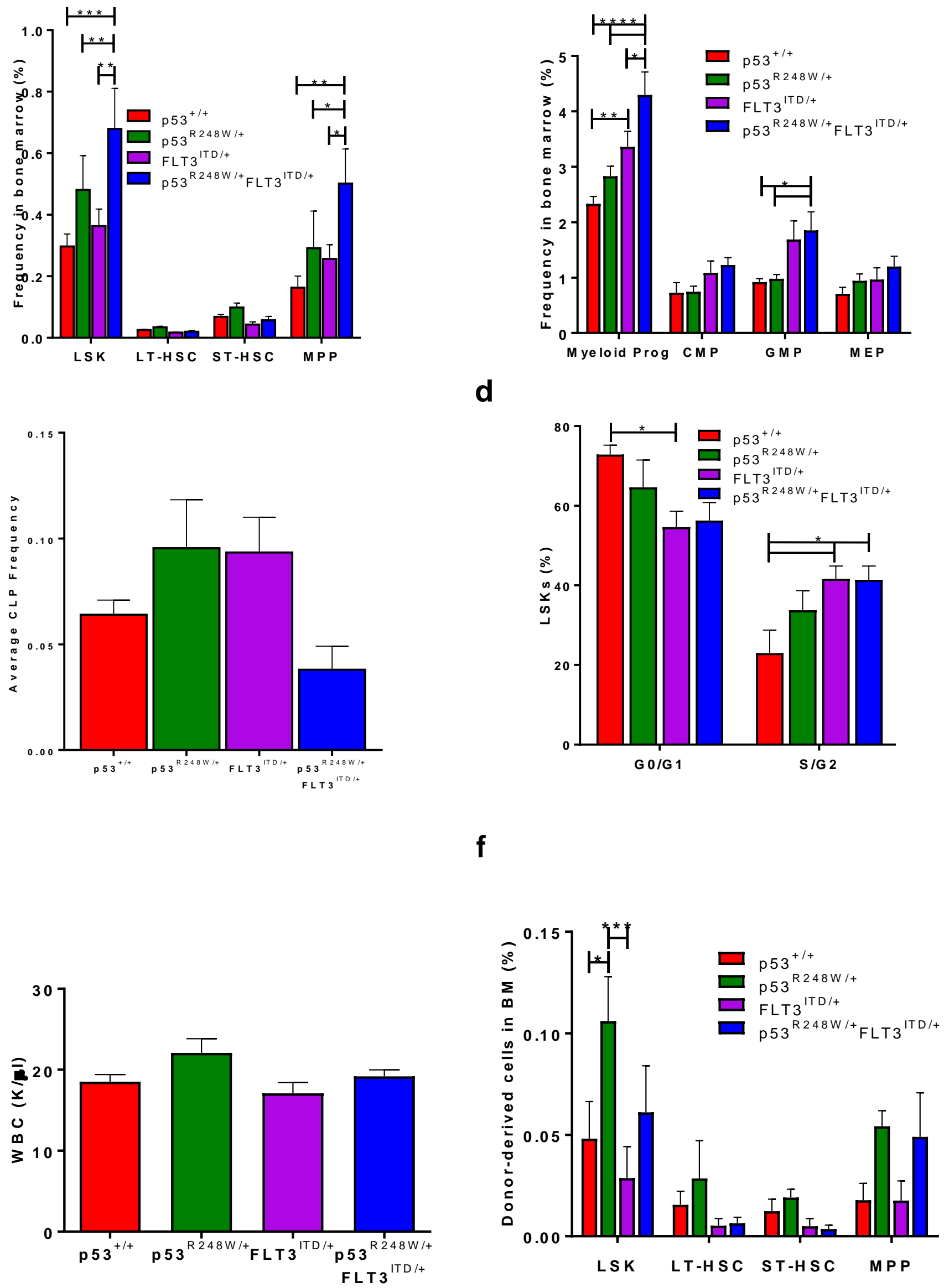

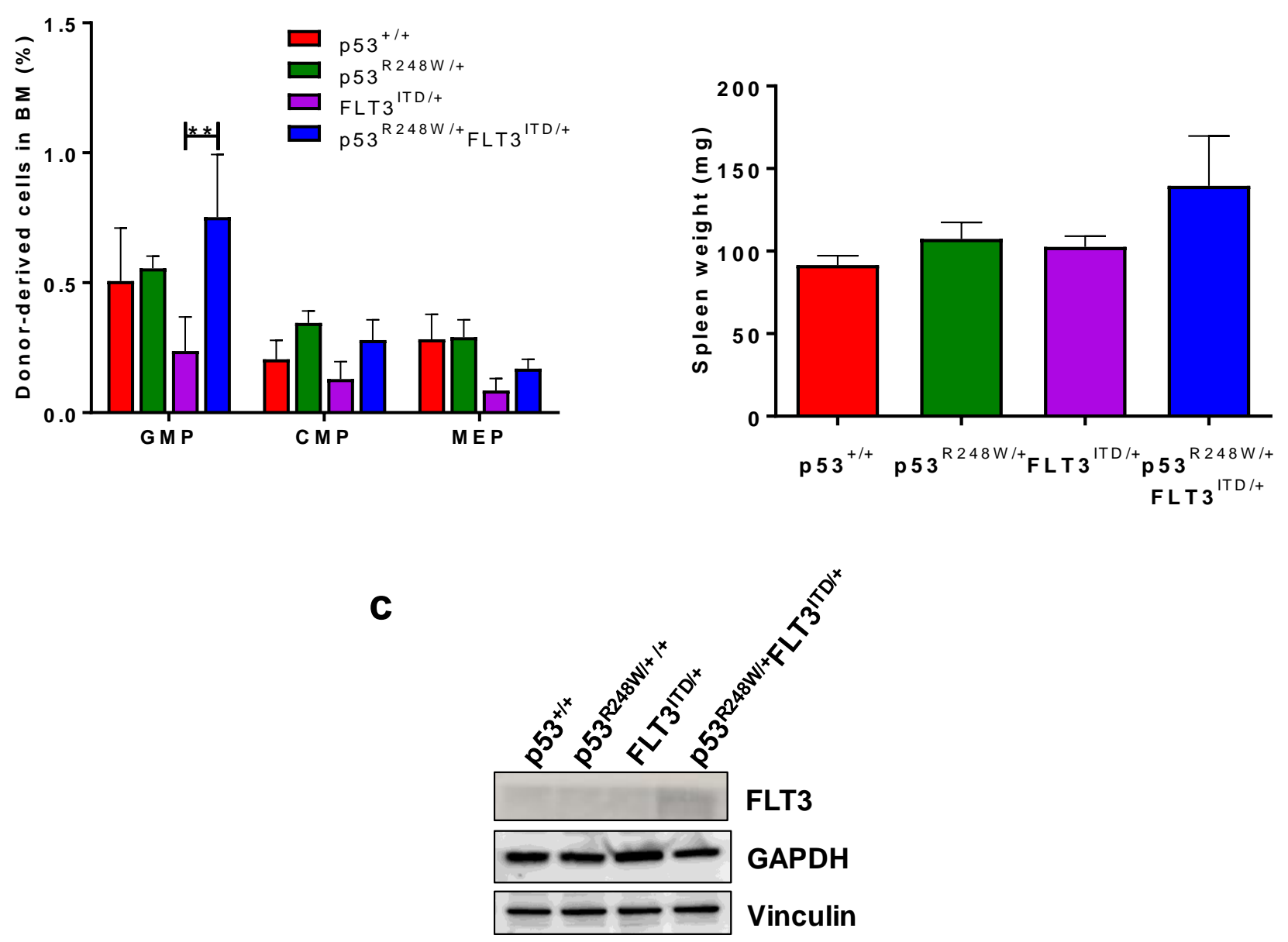

d

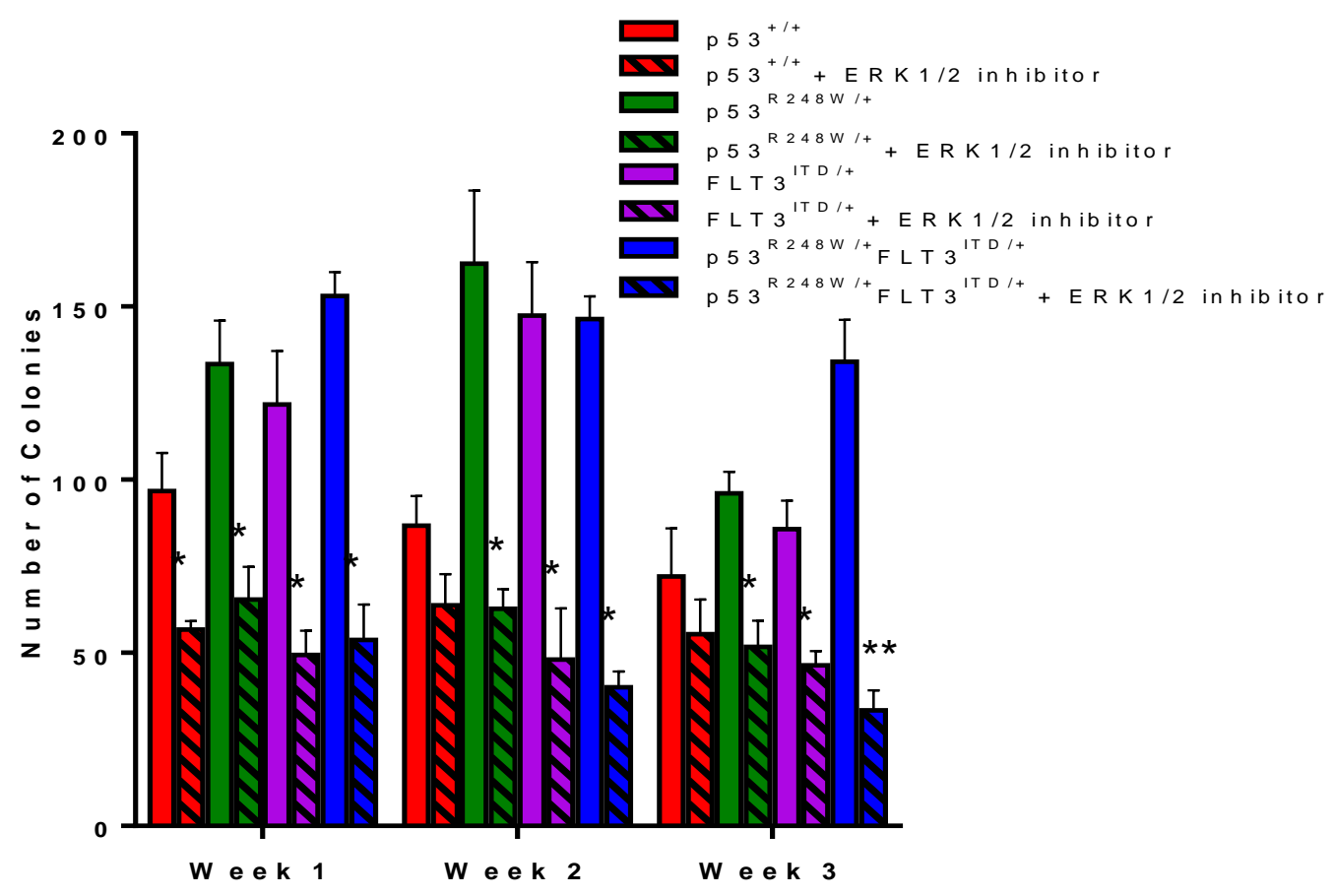

